\title{
CARCINOMA RENAL DE CÉLULAS CLARAS. ANÁLISIS DE INCIDENCIA Y SUPERVIVENCIA EN UN ÁREA SANITARIA DE 90,000 HABITANTES.
}

\author{
Daniel Santos Arrontes, Fernando de Castro Barbosa, María Paz Valer López-Fando', Manuel \\ Fernández Arjona e lgnacio Cortés Aránguez.
}

Servicio de Urología. Hospital Santa Bárbara. Puertollano. Ciudad Real. España.

Práctica privada'.

\begin{abstract}
Resumen.- OBJETIVOS: Evaluar los datos epidemiológicos y de supervivencia más relevantes del carcinoma renal de células claras (CCR) en un área geográfica de 90,000 habitantes, así como su asociación a factores de riesgo como el tabaco o el uso de diuréticos.
\end{abstract}

MÉTODOS: Se incluyeron todos los pacientes con diagnóstico histológico de carcinoma renal de células claras, entre el 1 de Enero de 1987 y el 31 de Enero de 2004. Se calcularon las tasas de incidencia ajustada (estandarizada) por 100.000 habitantes y año en el área sanitaria VIII de Ciudad Real (90.000 habitantes), el incremento porcentual anual y el índice epidémico. Para el ajuste poblacional se utilizaron los datos publicados por el Instituto Nacional de Estadística. Se evaluarán las diferencias de mortalidad entre ambos sexos, así como en función del consumo de tabaco, diuréticos o ambos, mediante el test de chi-cuadrado, o test exacto de Fischer,y la supervivencia global mediante un modelo de regresión de Cox.

RESULTADOS: Se diagnosticaron 38 CCR, con una incidencia máxima de 7,21 casos por 100,000 habitantes y año. Se produjo un incremento porcentual anual durante todo el periodo de un 2,06\%. No existieron diferencias estadísticamente significativas en la mortalidad asociada al sexo $(p=0,1)$, estadio tumoral $(p=0,063)$, consumo de tabaco $(p=0,13)$, diuréticos $(p=0,69)$ o ambos $(p=0,5)$. La mortalidad fue significativamente superior durante los primeros 5 años tras la intervención quirúrgica.

CONCLUSIONES: La incidencia del CCR está aumentando de forma progresiva, y es de preveer que esta tendencia se mantenga al alza en los próximos años. La mortalidad global (por cualquier causa) asociada al CCR es superior los primeros 5 años tras el diagnósti$\mathrm{CO}$.

Palabras clave: Carcinoma células renales, Incidencia, epidemiología.

Summary.- OBJECTIVES: To evaluate the most relevant epidemiological and survival data of the renal cell carcinoma (RCC) in a 90.000 citizens geographic area, as well as its association with risk factors such as tobacco or diuretic drugs use.

METHODS: All patients with the pathologic diagnosis of renal cell carcinoma between January Ist 1987 and January $37^{\text {st }} 2004$ were included in the study. Adjusted incidence rates (standardized) per 100.000 inhabitants year, annual percentage increase, and epidemic index in the health-care area VIII in Ciudad Real 190.000 inhabitants) were calculated. For the population 
adjustment we used the data Published by the National Institute of Statistics. We evaluated the differences in mortality between males/females, as well as in relation to tobacco consumption, using the chi-square test or the Fisher exact test, and the overall survival by a Cox regression model.

RESULTS: Thirty-eight RCC were diagnosed, with a maximum incidence of 7.21 cases per 100.000 inhabitants year. There was an annual percentage increase of $2.06 \%$ over the whole period. There were no statistically significant differences in gender-associated mortality $(p=0.1)$, tumor stage $(p=0.063)$, tobacco consumption ( $p=0.13$ ), diuretic drugs consumption ( $p$ $=0.69)$, or both $(p=0.5)$. Mortality was significantly higher during the first 5 years after surgery.

CONCLUSIONS: The incidence of RCC is progressively increasing, and it is predictable that this trend will be maintained in the next years. Overall mortality (any cause) associated with RCC is higher in the first five years after diagnosis.

Keywords: Renal cell carcinoma. Incidence. Epidemiology.

\section{INTRODUCCIÓN}

Los tumores renales constituyen aproximadamente el $3 \%$ de los tumores malignos. En el año 2002, en España fallecieron 1.471 pacientes por dicha causa, constituyendo únicamente el 1,5\% de la mortalidad oncológica (1). No obstante, su incidencia ha aumentado en todo el mundo, debido probablemente al desarrollo de técnicas de imagen, que se han asociado a un incremento de los tumores incidentales en pacientes asintomáticos.

Aunque una gran variedad de agentes, químicos y biológicos, han sido implicados en el desarrollo del cáncer renal, el conocimiento de su etiología no está del todo aclarado. En los últimos años se han desarrollado numerosos estudios poblacionales con la intención de clarificar estas causas (2). Dentro de las posibles etiologías se ha implicado al uso de diuréticos (3) o al consumo de tabaco (4).

El objetivo de este estudio es evaluar los datos epidemiológicos y de supervivencia más relevantes del carcinoma renal de células claras (CCR) en un área geográfica de 90.000 habitantes. Además se valorará la supervivencia en los pacientes fumadores, en aquellos que presentaban un tratamiento con diuréticos, o bien ambos factores. Además también se valorará la supervivencia global en relación al estadio tumoral y sintomatología al diagnóstico (hematuria micro o macroscópica, hallazgo incidental o cólico renoureteral).

\section{MATERIAL Y MÉTODOS}

En este estudio retrospectivo se incluyeron todos los pacientes con diagnóstico histológico de carcinoma renal de células claras, entre el 1 de Enero de 1987 y el 31 de Enero de 2004. Todos los casos fueron estadiados de acuerdo con la clasificación TNM de la International Union Against Cancer (UICC) correspondiente al año 2002 (5).

Se calcularon las tasas de incidencia ajustada (estandarizada) por 100.000 habitantes y año en el área sanitaria VIII de Ciudad Real $(90,000$ habitantes), el incremento porcentual anual (APC) y el índice epidémico (IE)- a partir de los datos de incidencia ajustada- (definido como el número de casos existentes anualmente dividido entre la mediana del número de casos presentes en el quinquenio anterior). Valores del índice epidémico superiores a 1,25 reflejan un incremento de la incidencia superior al esperado.

Para el ajuste poblacional se utilizaron los datos publicados por el Instituto Nacional de Estadística (INE) (1).

Se evaluarán las diferencias de mortalidad entre ambos sexos, así como en función del consumo de tabaco, diuréticos o ambos, mediante el test de chi-cuadrado o, en su lugar, el test exacto de Fischer cuando proceda. Al mismo tiempo se realizará un estudio de supervivencia global mediante un modelo de regresión de Cox. Con el fin de determinar el periodo de mayor mortalidad se calculará la densidad de probabilidad mediante un modelo de supervivencia de Kaplan-Meyer.

Para la elaboración de los resultados de utilizó el software SPSS v12.0 para Windows, considerándose significativo un error alfa inferior a 0,05.

\section{RESULTADOS}

Se diagnosticaron 38 carcinomas renales de células claras durante todo el periodo, de los cuales 22 correspondieron a varones $(57,9 \%)$ y 16 a mujeres $(42,1 \%)$. El tamaño tumoral medio fue de $6,2 \mathrm{~cm}$ (con un error estandar de la media -EEM- de 2,4 cm). La edad media al diagnóstico fue de 68 años (EEM 9,5 años). 
Los datos de incidencia absoluta, incidencia ajustada e índice epidémico, quedan reflejados en la Tabla I. Durante todo el periodo se ha producido un incremento de la incidencia de un 2,06\% anual. Además, el IE ha resultado superior a 1,25 en tres de los últimos cuatro años.

En 24 pacientes ha sido posible establecer el motivo inicial de consulta: 10 tumores incidentales $(41,6 \%), 12$ debutaron con hematuria $(50 \%)$ y 2 con cólico renoureteral $(8,3 \%)$, de los cuales, 5 que presentaron hematuria y 1 de los que debutaron con cólico renoureteral fallecieron, no existiendo diferencias estadísticamente significativas en la mortalidad según la causa inicial del estudio $(p=0,56)$.

En cuanto a la clasificación TNM, se diagnosticaron: 13 estadios pTla $(34,2 \%$ del total de CCR, 1 exitus), 3 estadios $\mathrm{pT} 1 \mathrm{~b}(7,9 \%), 6$ estadios pT2 (15,7\%, 2 exitus), 12 estadios pT3a $(31,5 \%$,

TABLA I. INCIDENCIA ABSOLUTA (IA), INCIDENCIA AJUSTADA (I AJUSTADA) E ÍNDICE EPIDÉMICO. * * INCREMENTO PORCENTUAL ANUAL.

\begin{tabular}{|cccc|}
\hline AÑO & IA & I Ajustada & IE \\
\hline 1987 & 1 & 1.09 & \\
\hline 1988 & 1 & 1,1 & \\
\hline 1989 & 3 & 3,36 & $N D$ \\
\hline 1990 & 3 & 3,4 & \\
\hline 1991 & 1 & 1,15 & \\
\hline 1992 & 1 & 1,14 & 0,5 \\
\hline 1993 & 1 & 1,14 & 0,99 \\
\hline 1994 & 1 & 1,12 & 0,97 \\
\hline 1995 & 5 & 5,7 & 4,07 \\
\hline 1996 & 1 & 1,13 & 0,99 \\
\hline 1997 & 1 & 1,14 & 1 \\
\hline 1998 & 3 & 3,43 & 3 \\
\hline 1999 & 2 & 2,3 & 2 \\
\hline 2000 & 1 & 1,16 & 0,5 \\
\hline 2001 & 2 & 2,35 & 2,02 \\
\hline 2002 & 4 & 4,72 & 2,05 \\
\hline 2003 & 1 & 1,19 & 0,5 \\
\hline 2004 & 6 & 7,21 & 3,1 \\
\hline & 38 & $2,06 * *$ & \\
\hline & & & \\
\hline
\end{tabular}

5 exitus) y 4 estadios pT3b (10,5\%, 3 exitus, uno de ellos era pT3bN1). En cuanto al grado Furhäm, se identificaron 3 grados I $7,9 \%$ del total de CCR), 28 grados $\|(73,7 \%), 6$ grados $|1|(15,7 \%)$ y 1 caso de grado IV $(2,6 \%)$. No se objetivaron diferencias, aunque los resultados se encuentran cerca de la significación estadística, en la mortalidad global (no cáncer específica) entre los estadios pT1-2 y PT3-4 $(p=0,063)$ (Figuras 1 y 2$)$.

El tiempo medio de seguimiento fue de 4 años con un EEM de 4,4 años. La mediana de supervivencia para este periodo no ha sido alcanzada todavía, habiéndose producido un total de 11 fallecimientos (2 mujeres y 9 hombres), sin existir diferencias significativas entre ambos sexos $(p=0,1)$ (Figura 3 ). Además de ello, tampoco existieron diferencias en la mortalidad asociada a pacientes fallecidos que consumían diuréticos ( 2 pacientes, $p=0,69)$, eran fumadores ( 1 paciente, $p=0,13$ ) o ambos ( 1 paciente, $p=0,5)$. La tasa de exitus fue superior durante los primeros 5 años tras el diagnóstico (densidad de probabilidad de 0,0004), reduciéndose significativamente a partir de dicho momento.

\section{DISCUSIÓN}

Respecto a los datos epidemiológicos puramente descriptivos, vemos que la incidencia del CCR es superior en varones, siendo la séptima década de la vida el momento de mayor incidencia, hallazgos ya demostrados por otros autores (6).

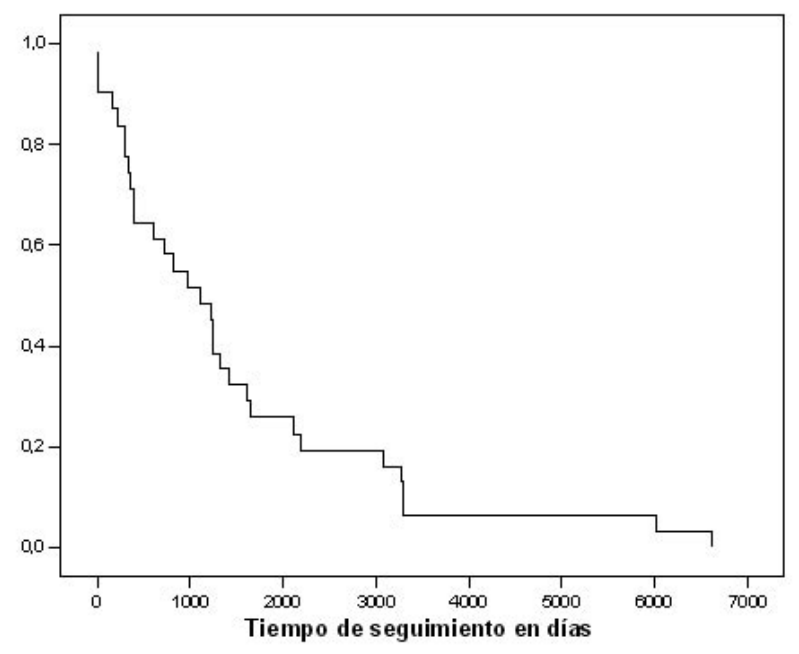

FIGURA 1. Supervivencia acumulada global no cáncer específica. El tiempo de seguimiento está expresado en días. 
Además los datos reflejan un incremento en la incidencia del CCR durante los últimos años. En tres de los últimos cuatro años, la incidencia ha sido superior a la esperada, con IE superiores al 1,25. Este hallazgo puede estar en relación con la implantación y uso intensivo de técnicas de imagen durante los últimos años en nuestro medio hospitalario. Este hecho, no sólo justifica este incremento, sino que su ausencia previa también podría ser la causa de que un $40 \%$ de los CCR diagnosticados correspondan a un estadio pT3 y que casi un $60 \%$ hayan sido diagnosticados de forma no incidental. Este hecho contrasta con los datos publicados en la literatura donde la gran mayoría de los tumores son incidentales y asintomáticos (7).

El tratarse de un área sanitaria predominantemente rural, con poca accesibilidad al centro hospitalario, con poblaciones del área sanitaria a más de $100 \mathrm{~km}$, también podrían justificar estos hallazgos. Del mismo modo, los datos de incidencia ajustada son ligeramente inferiores a los registrados en otras áreas sanitarias (8). Los picos de incidencia registrados en 1995 y en el año 2004 son difíciles de explicar, aunque podrían estar en relación con un descenso de la población activa, trasladada a otras áreas sanitarias, persistiendo el grupo poblacional de mayor riesgo (jubilados).

Sin embargo, creemos que los datos de incidencia se ajustan fielmente a la realidad, puesto que, nuevamente, dadas las características de la población, en su mayoría jubilados o desempleados, con

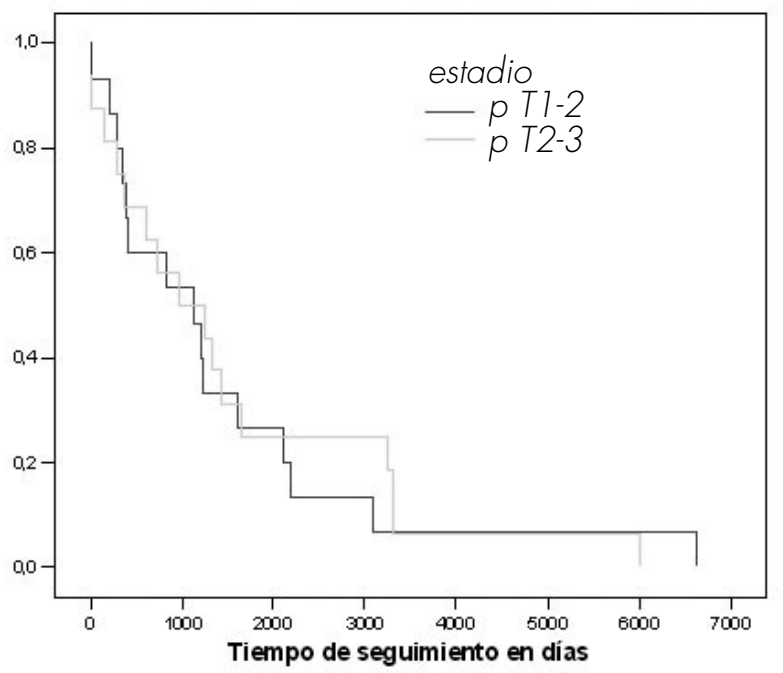

FIGURA 2. Supervivencia acumulada global no cáncer específica en función del estadio tumoral. Dado el escaso tamaño muestral se ha optado por expresar conjuntamente los estadios pT 1-2 y los pT3-4. Tiempo de seguimiento en días. un nivel adquisitivo medio-bajo, y la ausencia total de oferta privada en el área sanitaria limitan la pérdida de pacientes y su incorporación a otras áreas sanitarias.

En la interpretación del análisis de supervivencia que hemos presentado, no debemos concluir que el estadio tumoral no influya en la mortalidad. Está demostrado, y hay múltiples estudios que así lo corroboran, que el estadio local es el principal factor pronóstico en los pacientes con CCR (6). En este caso la ausencia de diferencias estadísticamente significativas entre ambos grupos se debe a dos factores fundamentales. En primer lugar, nos estamos refiriendo a datos de mortalidad global, no cáncer específica, y en segundo lugar, el tamaño muestral no nos permite tomar como válida esta afirmación. Sin embargo, si que resulta interesante, que la mortalidad en los pacientes sometidos a nefrectomía radical por CCR, es superior (por cualquier causa) en los primeros 5 años tras la cirugía. Esto puede ser un factor a tener en cuenta, sobre todo en pacientes con diagnóstico de novo de CCR, con edad avanzada o con una comorbilidad elevada.

Por otro lado, el consumo de tabaco, diuréticos o ambos, no afectan a la mortalidad global en pacientes con CCR. Aunque ambos son factores de riesgo reconocidos para el desarrollo del $\operatorname{CCR}$ (9, $10,11)$, no parecen relacionarse con una mayor o menor mortalidad. No obstante, su influencia sobre la incidencia del CCR que nos permitiría la elaboración de una odds-ratio asociada a la exposición, no

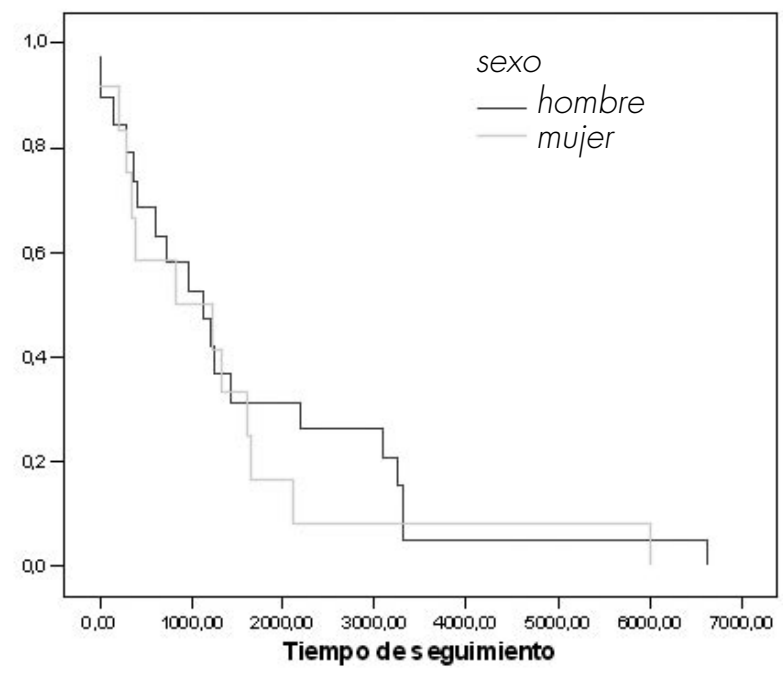

FIGURA 3. Supervivencia acumulada no cáncer específica en función del sexo. Tiempo de seguimiento en días. 
puede ser calculada mediante el planteamiento del presente estudio, sin introducir importantes errores metodológicos. Por dicho motivo, y de nuevo por el escaso tamaño muestral, tampoco debemos concluir que dichos factores de riesgo no afectan a la mortalidad, global o cáncer específica.

\section{CONCLUSIONES}

1. La incidencia del CCR está aumentando de forma progresiva, y es de preveer que esta tendencia se mantenga en los próximos años. El control de los factores de riesgo ya conocidos y la identificación de los factores aún desconocidos, debe intensificarse para modificar esta tendencia al alza.

2. La mortalidad global (por cualquier causa) asociada al CCR es superior los primeros 5 años tras el diagnóstico. No obstante, se requieren estudios prospectivos randomizados que confirmen estos hallazgos.

\section{BIBLIOGRAFÍA y LECTURAS RECOMENDADAS (*lectura de interés $y^{* *}$ lectura fundamental)}

*1. www.ine.es. Última entrada 11/8/2005.

**2. MURAI, M.; OYA, M.: "Renal cell carcinoma: etiology, incidence and epidemiology". Curr. Opin. Urol., 14: 229, 2004.
3. MESSERLI, F.H.: "Risk factors for renal cell carcinoma: Hypertension or diuretics?". Kidney Int., 67: 774, 2005.

*4. HUNT, J.D.; VAN DER HEL, O.L.; McMILLAN, G.P. y cols.: "Renal cell carcinoma in relation to cigarette smoking: meta-analysis of 24 studies". Int. J. Cancer, 114:101, 2005.

5. SOBIN, L.H.; WITTEKIND, C.H.: "TNM Classification of malignant tumours". International Union Against Cancer (UICC), Geneve. 2002.

6. GUDBJARTSSON, T.; HARDARSON, S.; PETURSDOTTIR, V. y cols.: "Histological subtyping and nuclear grading of renal cell carcinoma and their implications for survival: a retrospective nation-wide study of 629 patients". Eur. Urol., (En prensa) 2005.

7. LESLIE, J.A.; PRIHODA, T.; THOMPSON, I.M.: "Serendipitous renal cell carcinoma in the post-CT era: continued evidence in improved outcomes". Urol. Oncol., 21: 39, 2003.

8. SANTOS ARRONTES, D.; PÁEZ BORDA, A.; LUJÁN GALÁN, M. y cols.: "Incidencia del cáncer urológico en un área sanitaria de 300,000 habitantes". Actas Urol. Esp., 28: 646, 2004.

9. MOORE, L.E.; WILSON, R.T.; CAMPLEMAN, S.L.: "Lifestyle factors, exposures, genetic susceptibility, and renal cell cancer risk: a review". Cancer Invest., 23: 240, 2005.

10. FRYZEK, J.P.; POULSEN, A.H.; JOHNSEN, S.P. y cols.: "A cohort study of antihypertensive treatments and risk of renall cell cancer". Br. J. Cancer., 92: 1302, 2005.

11. HU, J.; UGNAT, A.M.: "Canadian Cancer Registries Epidemiology Research Group. Active and passive smoking and risk of renal cell carcinoma in Canada". Eur. J. Cancer., 41: 770, 2005 